\title{
Interstitial Ectopic Pregnancy Treated with Multi-Dose Methotrexate Protocol
}

\author{
Leah Roberts*, Arleen Ayala-Crespo, Juan Diaz Quinones \\ Department of Obstetrics, Gynecology and Reproductive Sciences, Temple University Hospital, Philadelphia, PA, USA \\ Email: ${ }^{\star}$ Leah.Roberts@tuhs.temple.edu
}

How to cite this paper: Roberts, L., Ayala-Crespo, A. and Quinones, J.D. (2019) Interstitial Ectopic Pregnancy Treated with Multi-Dose Methotrexate Protocol. Open Journal of Obstetrics and Gynecology, 9, 1527-1531.

https://doi.org/10.4236/ojog.2019.911148

Received: October 18, 2019

Accepted: November 16, 2019

Published: November 19, 2019

Copyright $\odot 2019$ by author(s) and Scientific Research Publishing Inc. This work is licensed under the Creative Commons Attribution International License (CC BY 4.0).

http://creativecommons.org/licenses/by/4.0/

\begin{abstract}
Interstitial ectopic pregnancies are very rare, however, they are extremely dangerous. Treatment consists of either surgical or medical management. This patient presented with no prior pregnancies, an inappropriately rising b-hCG, and eventually had ultrasound findings consistent with interstitial ectopic pregnancy. She was seen through the Emergency Department and had no insurance. She strongly desired to avoid surgery, and was successfully given a multi-dose regimen of methotrexate. Contraindication to methotrexate management includes an inability to follow-up, so a close therapeutic alliance was maintained to enable safe resolution of this case. She has since successfully carried an uncomplicated intrauterine pregnancy to term.
\end{abstract}

\section{Keywords}

Interstitial Pregnancy, Ectopic Pregnancy, Methotrexate

\section{Introduction}

The rate of ectopic pregnancy in the general population is $2 \%$ of all pregnancies. Risk factors for ectopic pregnancy include pelvic inflammatory disease, previous ectopic pregnancy, tubal surgery, assisted reproductive techniques (with two or more embryos transferred), and congenital uterine anomalies [1] [2]. Many of these risks factors are increasing in the general population as the rate of both sexually transmitted disease and assisted reproductive technologies rise. The only risk factor specific for interstitial ectopic pregnancy is ipsilateral salpingectomy [3]. Interstitial ectopic pregnancies have been found in at least 46 cases of ipsilateral salpingectomy, occurring between six months and thirteen years later [4]. Interstitial ectopic pregnancies account for only $2 \%-4 \%$ of all ectopic pregnancies. However, the risk of maternal mortality is approximately $2 \%-2.5 \%$, 
which is two to five times higher than other ectopic pregnancies [5] [6].

The interstitial portion of the fallopian tube is about $2 \mathrm{~cm}$ long and $0.7 \mathrm{~mm}$ wide and is highly vascular as it is located at the junction of the uterus and the proximal part of the fallopian tube [7]. It has abundant blood supply from both the uterine and ovarian vessels and therefore rupture and hemorrhage are more likely to lead to mortality [5] [6]. These pregnancies also tend to be diagnosed at a later gestational age, between six and twelve weeks, as the blood supply and distensibility of the architecture can support growth for a longer period of time.

These pregnancies are sometimes incorrectly referred to as cornual pregnancies, a term which actually describes conceptions in patients with Mullerian abnormalities [6] [7]. These develop in the horn of a septate or bicornate uterus [8]. These cornual pregnancies do have a higher risk of miscarriage, however, are not ectopic pregnancies as they are implanted within the endometrial cavity.

Criteria for ultrasound diagnosis include an empty uterine cavity, a chorionic sac separate and at least $1 \mathrm{~cm}$ from the lateral edge of the uterine cavity, and a thin myometrial layer surrounding the gestational sac [6]. Increased usage of three-dimensional technology in the hands of well-trained practitioners may also assist in diagnosis [9]. Diagnostic laparoscopy can also provide a definitive diagnosis, by directly visualizing the location of the pregnancy, and treatment by removal at the same time. Angular pregnancy, where the pregnancy implants in the lateral angle of the uterine cavity, will show displacement of the round ligament, with the gestational swelling medial to the ligament, where an interstitial pregnancy will be lateral [10]. If the diagnosis is uncertain in a stable patient, MRI could also be considered.

This case report describes an interstitial ectopic pregnancy in a 19 yo G1P0 successfully treated with multi-dose treatment with IM methotrexate alternating with folinic acid rescue.

\section{Case Presentation}

A 19 yo G1P0 with an unremarkable medical history presented to the emergency department with mild cramping midline pelvic pain. She reported that she had been seen at a different emergency department for the same pain two days earlier and had been diagnosed with a pregnancy of unknown anatomic location with a b-hCG of $396 \mathrm{mIU} / \mathrm{mL}$. After follow-up blood tests she was called by that institution and told to go to the closest ED, as her b-hCG had not risen appropriately. We continued to follow her b-hCG, and once she was above the discriminatory zone $(2000 \mathrm{mIU} / \mathrm{mL})$, she was instructed to come in for an ultrasound.

When she presented, she had a b-hCG of $4316 \mathrm{mIU} / \mathrm{mL}$. A transvaginal ultrasound revealed an oval-shaped echogenic focus with central cystic area measuring $2 \mathrm{~cm}$ in the region of the right uterine cornua. The diagnosis of interstitial pregnancy was made, and she was admitted for management. She was counseled on all available treatment options including medical and surgical management with cornual wedge resection, however, she strongly desired to avoid surgical 
management. Several medical techniques were discussed, and a plan was made for Interventional Radiology (IR) guided injection of methotrexate. Unfortunately, she was not a candidate for IR injection as they were not able to obtain a safe percutaneous window due to the interposed urinary bladder and bowel.

At this point, protocols for medical treatment were explored to find the method that best served the patient's specific situation. She was given multi-dose treatment with IM methotrexate, alternating with folinic acid rescue. With the multi-dose regimen, $1 \mathrm{mg} / \mathrm{kg}$ methotrexate is given on days $1,3,5$ and 7 , alternating with folinic acid $0.1 \mathrm{mg} / \mathrm{kg}$ IM on days $2,4,6$ and 8 . Serum b-hCG levels were obtained on each day that she received a methotrexate dosage. On day 3 her b-hCG had increased to $4389 \mathrm{mIU} / \mathrm{mL}$, day 5 was $4227 \mathrm{mIU} / \mathrm{mL}$, and day 7 was $3674 \mathrm{mIU} / \mathrm{mL}$ (13\% decrease), she was then followed weekly until complete resolution. Week one decreased appropriately to $1381 \mathrm{mIU} / \mathrm{mL}$, and it took almost one additional month to reach zero.

One challenge to this case was that the patient was uninsured, so therefore this medication had to be given through the emergency department. Contraindication to methotrexate management includes an inability to follow-up, so a close therapeutic alliance was maintained to enable safe resolution of this case. Pregnancy hormone levels were followed closely during treatment, where they were shown to decrease until complete resolution. Of note, one year after resolution of the interstitial pregnancy, the patient had an intrauterine pregnancy and term delivery without any complications at an outside institution.

\section{Discussion}

This case presented a challenge in balancing of patient autonomy and patient safety as well as avoiding paternalism. The sensitive part of this case was the patients firm desire to avoid surgery at all costs, and her desire for a future vaginal delivery, even with extensive discussion about risk of death if rupture should occur. This risk is a concern during labor whether the interstitial pregnancy is treated with medicine or surgery [11]. Extensive time in counseling was taken to ensure medical literacy in her choice to avoid surgery, as well as adequate understanding of risks of various management choices.

Our biggest limitation to the multi-dose regimen in this case was follow-up, as the patient was uninsured requiring follow up for lab work through the ED instead of clinic. The most important thing we did in this case was extensive counseling, and having strong support of her family. With her permission and desire, we were able to utilize her resources including her mother, sisters, and significant other, in order to enable adequate follow-up. In a patient who had previously had issues with follow-up, we had to ensure compliance to treatment and monitoring in order to agree to methotrexate treatment, as inability to follow-up is an absolute contraindication to medical management.

Although in the past we have used certified letters through the mail, in this patient both phone calls and SMS messages were much more successful in en- 
suring she attended her follow-up appointments. She was extremely compliant with her care immediately following her diagnosis and adequately followed up with us for the next year both in the ED, through telephone, and in our clinic once she was able to sign up for insurance through our assistance program. Interestingly, while this patient has since successfully carried a pregnancy to term, she continued to seek care at several institutions in the area throughout her pregnancy.

\section{Conclusion}

Interstitial ectopic pregnancies provide a significant challenge both in diagnosis and treatment. Many different regimens have been tried in order to best treat these pregnancies, however, there is no definitive way that works for all cases. Those cases that are stable may be well served by medical management with methotrexate through one of the options presented. These cases still pose a higher maternal mortality risk than other ectopic pregnancies, and require a close therapeutic relationship between patient and practitioner, as compliance with follow-up is integral to successful treatment.

\section{Authors' Contribution}

Leah Roberts: Conception of case report; Literature search; Analysis of clinical case; Drafting of manuscript.

Arleen Ayala-Crespo: Review for academic context; Critical revisions.

Juan Diaz Quinones: Review for academic context; Critical revisions.

\section{Conflicts of Interest}

The authors declare that there are no conflicts of interest regarding the publication of this paper nor do they have any funding to disclose.

\section{References}

[1] (2013) Ectopic Pregnancy. In: Cunningham, F., Leveno, K., Blood, S., Spong, C., Dashe, J., Hoffman, B., Casey, B. and Sheffield, J., Eds., Williams Obstetrics, Vol. 25, McGraw Hill, New York, 371-384.

[2] Faraj, R. and Steel, M. (2007) Management of Cornual (Interstitial) Pregnancy. The Obstetrician \& Gynaecologist, 9, 249-255. https://doi.org/10.1576/toag.9.4.249.27355

[3] Farquhar, C.M. (2005) Ectopic Pregnancy. The Lancet, 366, 583-591. https://doi.org/10.1016/S0140-6736(05)67103-6

[4] Grindler, N.M., Ng, J., Tocce, K. and Alvero, R. (2016) Considerations for Management of Interstitial Ectopic Pregnancies: Two Case Reports. Journal of Medical Case Reports, 10, 106. https://doi.org/10.1186/s13256-016-0892-9

[5] Izquierdo, L.A. and Nicholas, M.C. (2003) Three-Dimensional Transvaginal Sonography of Interstitial Pregnancy. Journal of Clinical Ultrasound, 31, 484-487. https://doi.org/10.1002/jcu.10201

[6] Kalidindi, M., Shahid, A. and Odejinmi, F. (2015) Expect the Unexpected: The Di- 
lemmas in the Diagnosis and Management of Interstitial Ectopic Pregnancy-Case Report and Literature Review. Gynecology and Minimally Invasive Therapy, 5, 35-37. https://doi.org/10.1016/j.gmit.2015.09.006

[7] Lau, S. and Tulandi, T. (1999) Conservative Medical and Surgical Management of Interstitial Ectopic Pregnancy. Fertility and Sterility, 72, 207-215.

https://doi.org/10.1016/S0015-0282(99)00242-3

[8] Malinowski, A. and Bates, S.K. (2006) Semantics and Pitfalls in the Diagnosis of Cornual/Interstitial Pregnancy. Fertility and Sterility, 86, 1764.e1711-1764. https://doi.org/10.1016/j.fertnstert.2006.03.073

[9] Perkins, K.M., Boulet, S.L., Kissin, D.M., Jamieson, D.J. and Group, N.A.S.N. (2015) Risk of Ectopic Pregnancy Associated with Assisted Reproductive Technology in the United States, 2001-2011. Obstetrics \& Gynecology, 125, 70-78.

https://doi.org/10.1097/AOG.0000000000000584

[10] Simpson, J.W., Alford, C.D. and Miller, A.C. (1961) Interstitial Pregnancy Following Homolateral Salpingectomy. A Report of 6 New Cases and Review of the Literature. American Journal of Obstetrics \& Gynecology, 82, 1173-1179. https://doi.org/10.1016/S0002-9378(16)36210-X

[11] Surbone, A., Cottier, O., Vial, Y., Francini, K., Hohlfeld, P. and Achtari, C. (2013) Interstitial Pregnancies' Diagnosis and Management: An Eleven Cases Series. Swiss Medical Weekly, 143, w13736. https://doi.org/10.4414/smw.2013.13736 